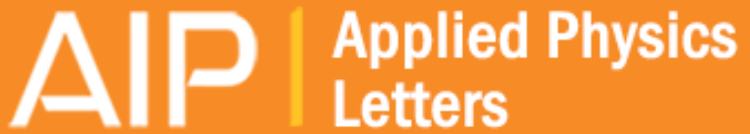

Intense optical second harmonic generation from centrosymmetric nanocrystalline para-nitroaniline

Dmitry V. Isakov, Michael S. Belsley, Etelvina de Matos Gomes, Hugo Gonçalves, Peter Schellenberg, and Bernardo G. Almeida

Citation: Applied Physics Letters 104, 181903 (2014); doi: 10.1063/1.4875908

View online: http://dx.doi.org/10.1063/1.4875908

View Table of Contents: http://scitation.aip.org/content/aip/journal/apl/104/18?ver=pdfcov

Published by the AIP Publishing

\section{Articles you may be interested in}

Solvent dependence of solvatochromic shifts and the first hyperpolarizability of para-nitroaniline: A nonmonotonic behavior

J. Chem. Phys. 119, 4409 (2003); 10.1063/1.1594181

Microscopic calculation of surface-induced second-harmonic generation in crystals of para-nitroaniline J. Chem. Phys. 112, 6757 (2000); 10.1063/1.481251

Solvent induced polarizabilities and hyperpolarizabilities of paranitroaniline studied by reaction field linear response theory

J. Chem. Phys. 100, 8240 (1994); 10.1063/1.466767

Optical second harmonic generation in Langmuir-Blodgett films of ndocosyl2methyl4nitroaniline Appl. Phys. Lett. 61, 1616 (1992); 10.1063/1.107511

Second harmonic generation of 2 methyl4nitroaniline by a neodymium: yttrium aluminum garnet laser with a tapered slabtype optical waveguide

Appl. Phys. Lett. 45, 333 (1984); 10.1063/1.95259

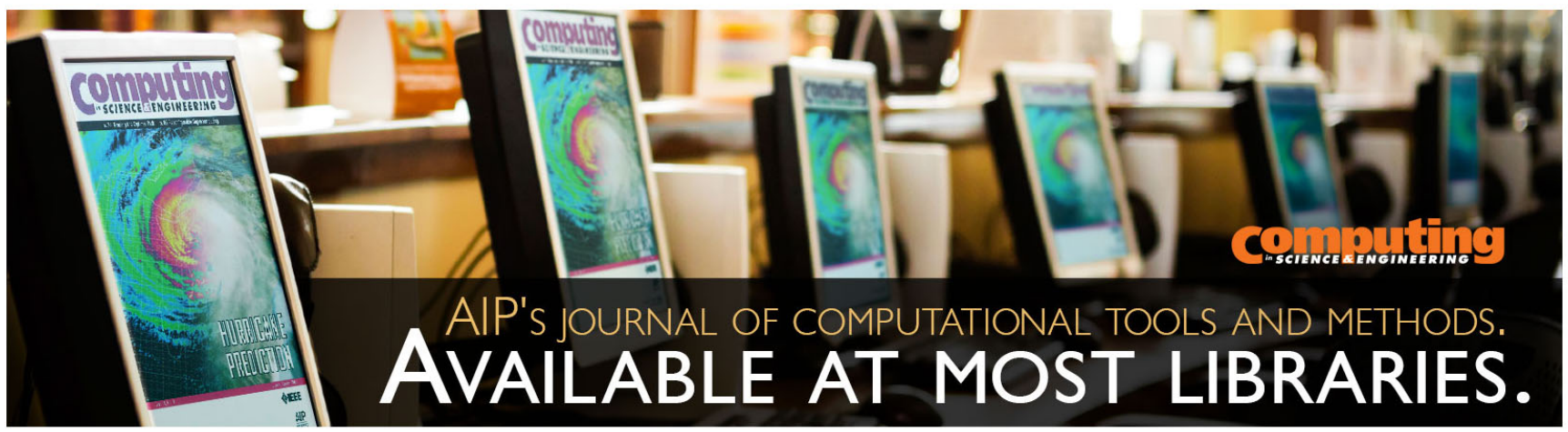




\title{
Intense optical second harmonic generation from centrosymmetric nanocrystalline para-nitroaniline
}

\author{
Dmitry V. Isakov, ${ }^{1,2, a)}$ Michael S. Belsley, ${ }^{1}$ Etelvina de Matos Gomes, ${ }^{1}$ Hugo Gonçalves, ${ }^{1}$ \\ Peter Schellenberg, ${ }^{1}$ and Bernardo G. Almeida ${ }^{1}$ \\ ${ }^{1}$ Center of Physics, University of Minho, Campus de Gualtar, 4710-057 Braga, Portugal \\ ${ }^{2}$ Department of Materials, University of Oxford, Parks Road, Oxford OX1 3PH, United Kingdom
}

(Received 25 March 2014; accepted 27 April 2014; published online 8 May 2014)

\begin{abstract}
We demonstrate an approach that uses normally centrosymmetric, but highly polarizable organic molecules of para-nitroaniline ( $p$-NA) as very efficient second harmonic generator (SHG) for near infra-red light. The approach is based on an effective manipulation of the nanocrystalline size and their assembly into a highly orientated mesocrystalline structures. The resulting mesocrystalline form of $p$-NA consists of multiple nanocrystals with common orientation, providing a set of surfaces with highly aligned molecules forming a head-to-tail polar arrangement. The second harmonic generation efficiency from this arrangement of mesocrystalline nanostructures is comparable to that from the conventional non-centrosymmetric organic crystals. The experimental results indicate that a very strong second-order nonlinear response can be obtained from small D- $\pi$-A organic molecules with elevated molecular hyperpolarizabilities even if these molecules tend to crystallize in centrosymmetric structures. (c) 2014 AIP Publishing LLC. [http://dx.doi.org/10.1063/1.4875908]
\end{abstract}

Organic materials formed by push-pull $\pi$-conjugated molecules inherently possess very high values of molecular second order polarizability, making them very efficient generators of second harmonic light on an individual molecular scale. ${ }^{1}$ However, the application of such materials for light wavelength conversion has been rather limited since almost all linear push-pull molecular systems crystallize in a centrosymmetric structures in which the individual molecular second harmonic responses destructively interfere. Furthermore, it has turned out to be difficult to grow high optical quality crystals for the few organic molecular systems which crystallize in a noncentrosymmetric structure. On the other hand, even centrosymmetric crystals possess a local symmetry breaking at their surfaces, which in bulk crystals can generate a small amount of second harmonic light. ${ }^{2,3}$ Also, polar effects, such as pyroelectricity, can be obtained on surfaces of certain centrosymmetric molecular crystals due to growth-induced polarity formation. $^{4,5}$ A surface quadratic susceptibility up to 5 $\times 10^{-22} \mathrm{~m}^{2} / \mathrm{V}$ has been predicted for centrosymmetric molecular para-nitroaniline ( $p$-NA) crystal, ${ }^{6}$ and previously an inhomogeneous second harmonic generation (SHG) has been observed on the cleavage surface of $p$-NA crystals with intense spots generated at etch pits. ${ }^{7,8}$ We demonstrate here that it is possible to generate appreciable amounts of second harmonic light from highly oriented $p$-NA nanocrystals embedded within electrospun polymeric nanofibers.

Para-nitroaniline $\left(\mathrm{C}_{6} \mathrm{H}_{6} \mathrm{~N}_{2} \mathrm{O}_{2}\right)$ is a paradigmatic molecular building block for organic nonlinear optics. It has a delocalized $\pi$-electron system and unsaturated bridge linking a donor amino group $\left(\mathrm{NH}_{2}\right)$ and an acceptor nitro group $\left(\mathrm{NO}_{2}\right)$. Delocalization of the $\pi$ electrons leads to an asymmetric charge distribution resulting in a strong molecular optical nonlinearity. ${ }^{9}$ Endowed with an already large electric dipole moment in the ground electronic state $\left(\mu_{g}=6.2 \mathrm{D}\right.$ in

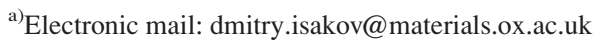

dioxane), upon $\pi-\pi^{*}$ photo-excitation, the molecular dipole moment is increased almost two-fold as a considerable amount of charge is transferred from the donor to the acceptor group resulting in a large molecular first hyperpolarizability $\beta{ }^{10-12}$ The induced transition dipole is along the charge-transfer axis of the molecule which points from the donor to the acceptor groups. Despite this exceptional molecular nonlinear optical response, $p$-NA crystallizes in a centrosymmetric space group $\mathrm{P} 21 / \mathrm{n}$, which impedes the observation of any macroscopic second-order optical effect. ${ }^{13}$

Here, we show that it is possible to overcome this restriction by embedding the molecules into electrospun fibers and demonstrate an approach that allows centrosymmetric nanocrystals as highly efficient optical frequency converter. We accomplish this through an effective manipulation of the $p$-NA nanocrystalline surface-to-volume ratio and their assemblage into highly oriented mesocrystalline structures (this structure is an intermediate between a single crystal and unoriented polycrystals) ${ }^{14,15}$ with a dominant acentric surface. This has been accomplished by the inclusion of $p$-NA into aligned arrays of electronspun polymer fibers.

Electrospinning is a nanofabrication technique used to produce nanofibers from a variety of materials, where a high voltage is applied to a viscous polymer solution on a sharp conducting tip, causing it to form a Taylor cone and a fluid jet is extracted from the cone and accelerated toward a grounded collecting substrate. The fibers solidify extremely rapidly due to in-flight solvent evaporation. The polymer nanofibers are formed by the creation and associated elongation of an electrified fluid jet, which causes local poling and by consequence enhanced properties. ${ }^{16,17}$ The electrospinning technique provides intrinsic spatial orientation of the embedded nanoparticles within the fibers. ${ }^{18}$ As shown previously, the size of the nanocrystals (and consequently their surface-to-volume ratio) can be efficiently regulated by controlling the fiber's diameter through an adjustment of the 
electrospinning processing parameters. ${ }^{19}$ Moreover, a mesocrystalline structure amongst the fibers can be obtained by ensuring an in-plane-oriented parallel arrangement of the nanofibers. ${ }^{20}$

As a consequence of the electrospinning process, $p$-NA is incorporated into poly(L-lactic acid) (PLLA) polymer fibers with a strongly preferred crystallographic orientation with respect to the fibers mat. The X-ray diffraction pattern, assigned to the centrosymmetric crystalline structure, is dominated by the Bragg reflection from the (202) plane (Figure 1(a)) which is two orders of magnitude more intense than all the other observed reflections although all reflections maybe assigned to the centric crystalline structure. We conclude that $p$-NA nanocrystals embedded within the polymer matrix are oriented with their (202) crystallographic plane parallel to the nanofibers array, forming a mesocrystalline structure. That plane, which happens to be parallel to the natural cleavage plane (101) in bulk crystals, cuts in between two molecules related to each other by a screw two-fold axis having its $\mathrm{CT}$ intra-molecular axis in that plane and parallel to the surface termination (Figure 1(b)). As a result, the crystal surface layer contains a head-to-tail polar arrangement of molecules with a maximum net transition dipole moment exhibited in that plane, which is essential to induce surface SHG in nanocrystalline $p$-NA. Indeed, the aligned assemblage of $p$-NA nanocrystals within the PLLA fiber mat was observed to be a strong generator of second harmonic light when illuminated by a Q-switched Nd:YAG laser emitting pulses at $1064 \mathrm{~nm}$. The green second harmonic light from a $p$-NA nanofibers mat is well seen by the naked eye. Nanofibers composed solely of PLLA do not generate any measurable amount of second harmonic light.
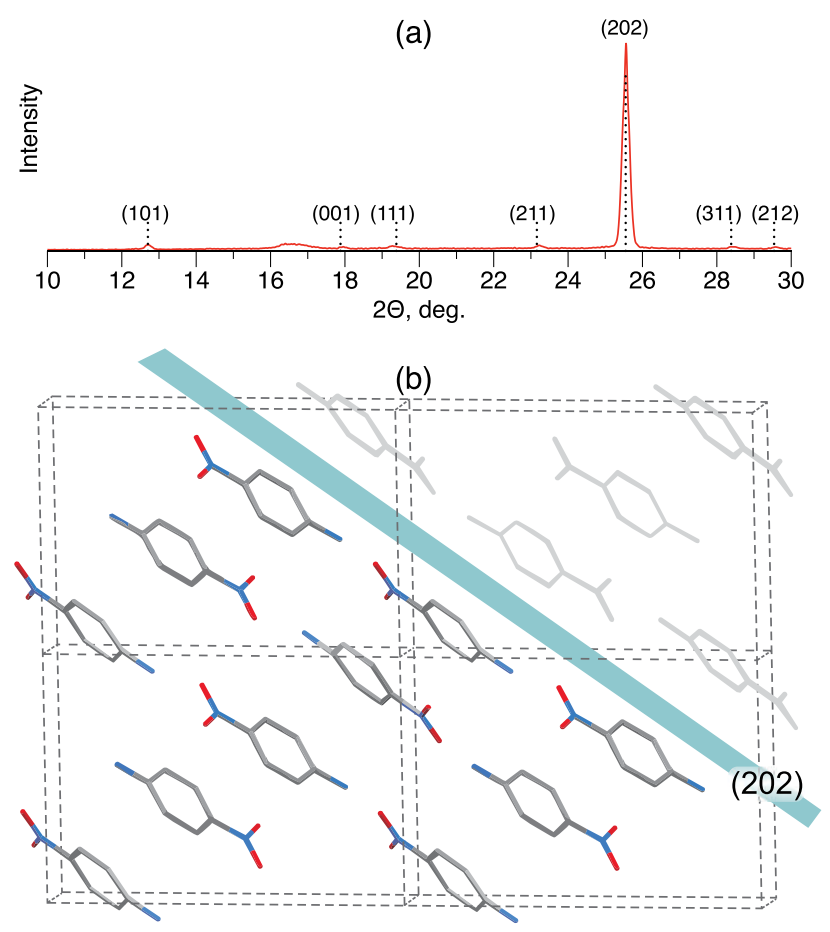

FIG. 1. (a) X-ray diffraction pattern revealing strong orientation observed in $p$-NA mesocrystalline structure produced by electrospinning. (b) Four unit cells of $p$-NA with shown (202) plane terminated the periodic crystal lattice by the surface.
To quantitatively estimate the effective nonlinear susceptibility of the $p$-NA mesocrystalline structure, a metanitroaniline $\left(\mathrm{C}_{6} \mathrm{H}_{6} \mathrm{~N}_{2} \mathrm{O}_{2}\right.$, mNA) oriented single crystal plate was used as a reference. The $p$-NA second harmonic intensity was measured in transmission relative to the mNA plate signal. The two molecules, mNA and $p$-NA, are isomeric but in mNA the donor $\left(\mathrm{NO}_{2}\right)$ group occupies the meta position on the benzene ring, which gives rise to a somewhat smaller molecular dipole moment and a lower molecular hyperpolarizability than in $p$-NA although within the same order of magnitude. ${ }^{21}$ However, mNA is an established nonlinear optical material which crystallizes in the polar space group $P b c 2_{1}$ with an acentric structure. ${ }^{22}$

For a mNA crystal oriented with its two-fold $(Z)$ polar axis parallel to the analyzer and polarizer axis, the SHG polarization $P_{z}^{2 \omega}=\epsilon_{0} d_{33} E_{z}^{\omega} E_{z}^{\omega}$ is also in the $Z$-axis direction and the SHG intensity is

$$
I_{m N A}^{2 \omega} \propto \frac{d_{33}^{2} l^{2}\left(I^{\omega}\right)^{2}}{n^{2 \omega}\left(n^{\omega}\right)^{2}} \operatorname{sinc} c^{2}\left(\frac{\Delta k L}{2}\right),
$$

where $\Delta k=2 \pi\left(n^{\omega}-n^{2 \omega}\right) / \lambda_{\text {inc }}$ is the phase mismatch, $L=10 \mu \mathrm{m}$ the crystal thickness, $n^{\omega}$ and $n^{2 \omega}$ the refractive indices for infrared and green light, respectively. ${ }^{23}$

On the other hand, for a $p$-NA fiber the generated SHG intensity would be similar to the above expression with the appropriate substitutions in the refractive indices, $d_{33}^{m N A}$ $\rightarrow d_{e f f}^{p-N A}$, and $L \rightarrow l$ the fiber thickness which is roughly $400 \mathrm{~nm}$ and setting the $\operatorname{sinc} c^{2}$ function to unity, since the coherence length is expected to be much greater than a given fiber dimension. Using the same incident intensity, the SHG response from mNA crystal was measured to be approximately 1000 times higher than that from a single $p$-NA fiber. Taking into account the vastly different areas illuminated by the incident beam in the two cases $\left(\pi D^{2} / 4\right.$ versus $D l$ where $D$ is the diameter of the incident focused laser beam $\approx 6 \mu \mathrm{m}$ ), we can estimate a value for the effective nonlinear susceptibility of the mesocrystalline $p$-NA fiber as

$$
d_{e f f}^{\mathrm{pNA}} \approx d_{33}^{\mathrm{mNA}} \frac{L}{l} \sqrt{\frac{D I_{\mathrm{pNA}}}{l I_{\mathrm{mNA}}}} \approx 2 d_{33}^{\mathrm{mNA}},
$$

where $I_{i}$ represents the signals measured from the mNA crystal plate and the $p$-NA fiber. Here, we neglected the effects of any possible phase mismatch in the mNA crystal plate as well as differences in the refractive indices and near unity numerical factors such as $\pi / 4$. This implies that the SHG response generated by a single $p$-NA nanofiber has an effective mean nonlinear optical coefficient $d_{\text {eff }}$ of the same order of magnitude as the highest nonlinear optical coefficient of an mNA crystal $d_{33}=49.2 \times 10^{-9} \mathrm{esu}^{22}$ that is $d_{\text {eff }}^{p-N A} \approx$ $2 d_{33}^{m N A} \approx 10^{-7}$ esu. This is probably a slight underestimate since the $p$-NA nanocrystals only occupy about half of the fibers volume and the coherence length of mNA is close to the thickness of the plate. It is also important to note that the signals generated from $p$-NA nanofibers are quite stable over time. Fibers that were kept at room temperature for over a year still generate signals at the same level as weekly prepared samples. This constancy is in sharp contrast to electric field poled $p$-NA molecules in polymer blends which tend to reorient themselves on a timescale of a few weeks. ${ }^{24}$ 
The observed SHG signal measured in $p$-NA embedded in the PLLA fibers is mainly a surface effect, as the nanocrystals structure keep their center of inversion. Detailed microscopic calculations on the surface quadratic susceptibility in $p$-NA for a (202) crystal plane surface termination predicted that for the dominate first surface layer contributions come from the tensor elements $\chi_{y y y}^{2 \omega}$ and $\chi_{x x y}^{2 \omega}$, with values corresponding to $6.57 \times 10^{-10}$ esu and $5.18 \times 10^{-10}$ esu, respectively (the axes are chosen in accordance with arrangement undertaken in Ref. 6, where $x$ and $y$ are taken to be in the plane of the fiber mat while and $z$ is normal to that plane). Figure 2 presents the second harmonic generation measured as a function of the polarization of the incident laser beam, which propagates normal to the fibers mat plane and therefore to (202) $p$-NA nanocrystals surfaces. The SHG signal obtained from $\phi-p$ and $\phi-s$ configurations (where $\phi$ indicates the polarization angle of the incident light relative to the fiber axis which varies from $0^{\circ}-360^{\circ}$ and $p$ and $s$ indicate the polarization of the detected second harmonic light parallel and perpendicular to the fiber axis, respectively), have a relative ratio of approximately 2 which is consistent with the theoretical calculations (1.73) for the static case (limit of zero frequency for the incident light) corresponding to the squared ratio for the above tensor elements. ${ }^{6}$

We also considered the possibility that the strong SHG obtained in the centrosymmetric nanostructure could originate from single polar $p$-NA molecules distributed with a strong preferential orientation within the fiber direction. This hypothesis basically assumes an oriented gas model when the overall SHG intensity of nanoparticles in the illuminated focal volume originates from the coherent sum of all the induced dipoles and is proportional to the square of the number of molecules. ${ }^{25}$ To empirically survey this assumption, fluorescence lifetime microscopy experiments have been carried out on layers of $p$-NA nanofibers (with an average diameter of roughly $430 \mathrm{~nm}$ ) as well as on $p$-NA bulk crystals and the decay times compared to those of the fluorescence lifetime decay of $p$-NA in solution. Figure 3 shows that the fluorescence lifetime decay in $p$-NA fibers as well as in the bulk crystal are fairly similar over the entire sample while the fluorescence lifetime in solution is dramatically different. This indicates that the $p$-NA molecules in a bulk crystal and (a)

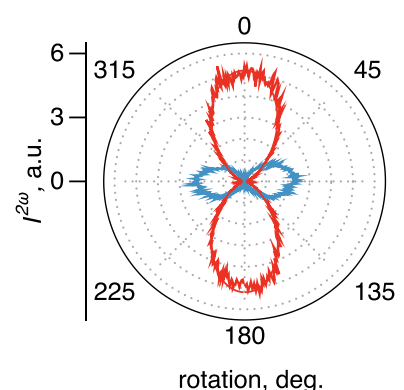

(b)

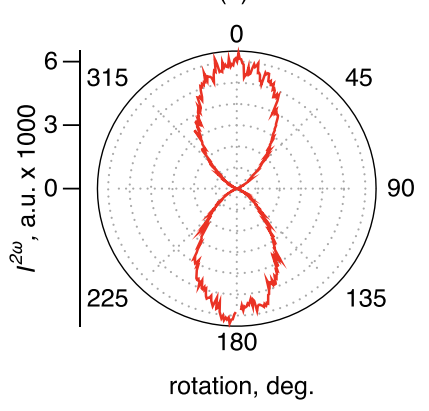

FIG. 2. Polarized SHG signal from a single $p$-NA nanofiber (a) and the $m$ NA single crystal used a reference (b). The red curve corresponds to $\varphi-p$ polarizations while the blue curves represents the $\varphi-s$ polarizations. Here, $q$ indicates the polarization angle of the incident light relative to the fiber axis which varies from $0^{\circ}-360^{\circ}$ and $p$ and $s$ indicate the polarization of the detected second harmonic light parallel and perpendicular to the fiber axis, respectively.
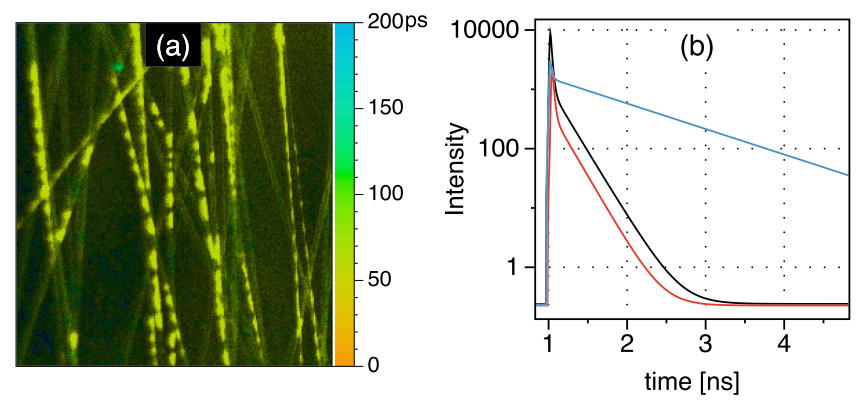

FIG. 3. (a) $50 \times 50 \mu \mathrm{m}$ micrograph of $p$-NA nanofibers obtained by Fluorescence lifetime Imaging Microscope and; (b) double exponential fluorescence decay obtained from $p$-NA single crystal (black curve), nanofibers (red curve) and solution (blue curve).

inside the fibers aggregate in similar structures with a strong exitonic coupling between individual molecular units.

A plausible mechanism for the strong SHG by $p$-NA fiber is that the mesocrystalline structure results from the confinement of well oriented $p$-NA nanocrystals embedded within the polymer fibers as an assembly of coherent nanosize surface sources with constructive interference between the propagating and induced nonlinear waves. Not only will the response of each individual nonlinear dipole add in phase with the contributions from the other dipoles within a given nanocrystal, but given that the fiber dimensions are much smaller than the coherence length, signals from different nanocrystals within the volume of the incident laser will emit second harmonic light coherently. In essence, they perform in the same manner as a poled doped polymer solution of $p$-NA, with the additional advantage that there is a single crystalline like structure, formed by all orientated hydrogen bonded molecules, embedded in nanocrystals where the surface effects are greatly enhanced. For these systems, phase matching becomes irrelevant which opens up the possibility of exploiting nominally non-phase matchable but large effective nonlinear susceptibilities in individual nanocrystals or even normally non-phase matchable materials. Furthermore, the polymer matrix could also play some role. Having a different refractive index from the $p$-NA nanocrystals the local electric field will be modified which can lead to enhancement of the second harmonic response. The theoretical calculations performed for surface quadratic susceptibility in pNA crystals ${ }^{6}$ assumed the bulk crystal to be infinite and to be placed in a vacuum and effects arising from the proximity of another dielectric interface were not considered.

To conclude, an extremely strong surface induced second harmonic generation has been observed from a mesocrystalline structure of centrosymmetric donor-acceptor organic molecules of para-nitroaniline consisting of aligned array of electrospun nanofibers. Our results demonstrate that it is possible to engineer a nanofiber array of centrosymmetric nanocrystals that have an SHG response with a similar magnitude to that of a single noncentrosymmetric organic crystals. This effect takes advantage of the multiple crystal surfaces having a common orientation with highly aligned molecules forming a head-to-tail dipolar arrangement.

This work was financially supported by the European Regional Development Fund (ERDF) through Programa Operacional Factores de Competitividade (COMPETE: 
FCOMP-01-0124-FEDER-014628) and the Portuguese Fundação para a Ciência e Tecnologia (PTDC/CT-NAN/ 114269/2009 and PTDC/CTM/105597/2008).

${ }^{1}$ D. S. Chemla and J. Zyss, Nonlinear Optical Properties of Organic Molecules (Academic Press, New York, 1987).

${ }^{2}$ P. C. Ray, Chem. Rev. 110, 5332 (2010).

${ }^{3}$ Y. R. Shen, Annu. Rev. Phys. Chem. 40, 327 (1989).

${ }^{4}$ C. Gervais, T. Wüst, N.-R. Behrnd, M. Wübbenhorst, and J. Hulliger, Chem. Mater. 17, 85 (2005).

${ }^{5}$ J. Hulliger, S. W. Roth, A. Quintel, and H. Bebie, J. Solid State Chem. 152, 49 (2000).

${ }^{6}$ M. Malagoli and R. W. Munn, J. Chem. Phys. 112, 6757 (2000).

${ }^{7}$ H. Kobayashi and M. Kotani, Mol. Cryst. Liq. Cryst. Sci. Technol., Sect. A 252, 277 (1994).

${ }^{8}$ H. Kobayashi and M. Kotani, Mol. Cryst. Liq. Cryst. Sci. Technol., Sect. A 278, 125 (1996).

${ }^{9}$ C. C. Teng and A. F. Garito, Phys. Rev. Lett. 50, 350 (1983).

${ }^{10}$ J. N. Woodford, M. A. Pauley, and C. Wang, J. Phys. Chem. A 101, 1989 (1997).

${ }^{11}$ S. P. Karna, P. N. Prasad, and M. Dupuis, J. Chem. Phys. 94, 1171 (1991).
${ }^{12}$ B. Champagne, Chem. Phys. Lett. 261, 57 (1996).

${ }^{13}$ K. N. Trueblood, E. Goldish, and J. Donohue, Acta Crystallogr. 14, 1009 (1961).

${ }^{14}$ R.-Q. Song and H. Cölfen, Adv. Mater. 22, 1301 (2010).

${ }^{15}$ L. Zhou and P. O'Brien, Small 4, 1566 (2008).

${ }^{16}$ D. Isakov, E. de Matos Gomes, I. Bdikin, B. Almeida, M. Belsley, M. Costa, V. Rodrigues, and A. Heredia, Cryst. Growth Des. 11, 4288 (2011).

${ }^{17}$ A. Camposeo, F. Di Benedetto, R. Cingolani, and D. Pisignano, Appl. Phys. Lett. 94, 043109 (2009).

${ }^{18}$ M. Richard-Lacroix and C. Pellerin, Macromolecules 46, 9473 (2013).

${ }^{19}$ D. Isakov, E. de Matos Gomes, M. S. Belsley, B. Almeida, and N. Cerca, Nanoscale 4, 4978 (2012).

${ }^{20}$ D. V. Isakov, E. de Matos Gomes, L. G. Vieira, T. Dekola, M. S. Belsley, and B. G. Almeida, ACS Nano 5, 73 (2011).

${ }^{21}$ P. D. Southgate and D. S. Hall, Appl. Phys. Lett. 18, 456 (1971).

${ }^{22}$ P. D. Southgate and D. S. Hall, J. Appl. Phys. 43, 2765 (1972).

${ }^{23}$ A. Yariv and P. Yeh, Optical Waves in Crystals: Propagation and Control of Laser Radiation (John Wiley, New York, 1984).

${ }^{24}$ T. Miyazaki, T. Watanabe, and S. Miyata, Jpn. J. Appl. Phys., Part 2 27, L1724 (1988).

${ }^{25}$ J. Zyss and J. L. Oudar, Phys. Rev. A 26, 2028 (1982). 